\title{
AIAA 2004-1233
}

\section{Detached-Eddy Simulation of Slat and Flap Aerodynamics for a High-Lift Wing}

Russell M. Cummings \& Scott A. Morton

Department of Aeronautics

United States Air Force Academy

USAF Academy, CO 80840

James R. Forsythe Cobalt Solutions, LLC Springboro, $\mathrm{OH} 45504$

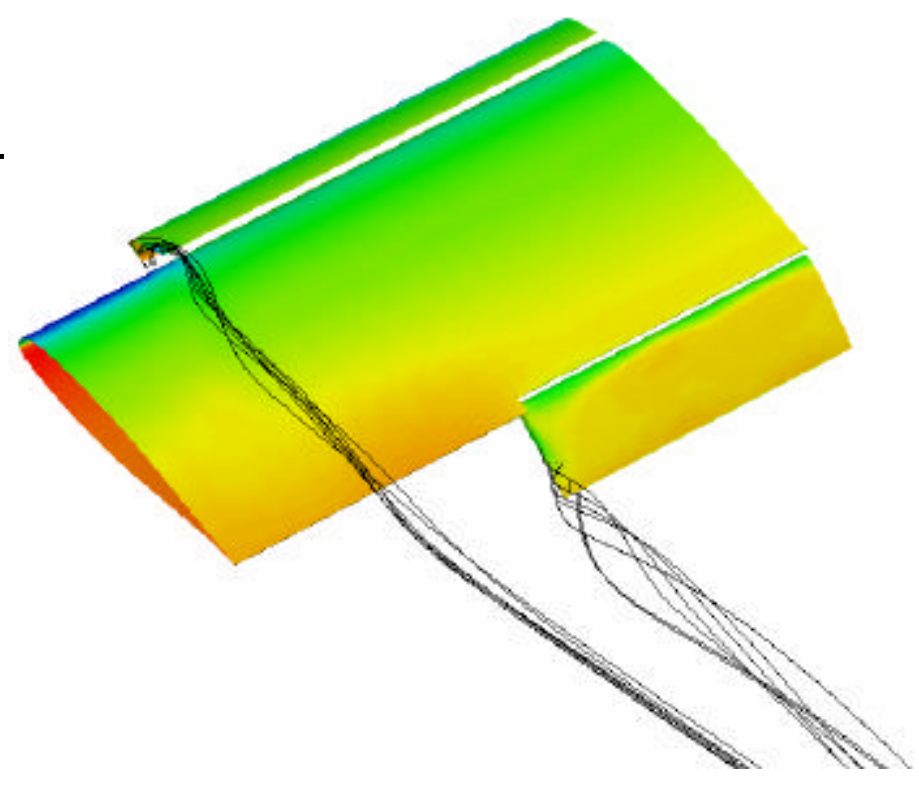

\section{$42^{\text {nd }}$ Aerospace Sciences Meeting 5-8 January 2004 Reno, Nevada}




\title{
DETACHED-EDDY Simulation OF Slat ANd FlaP AERODYNAMICS FOR A HIGH-LIFT WING
}

\author{
Russell M. Cummings, ${ }^{*}$ Scott A. Morton** \\ Department of Aeronautics, USAF Academy, CO 80840 \\ $\&$ \\ James R. Forsythe ${ }^{\dagger}$ \\ Cobalt Solutions, LLC, Springboro, OH 45504
}

\begin{abstract}
Three-dimensional multi-element wings are simulated to investigate slat and flap aerodynamics using Detached-Eddy Simulation. The computations are performed by solving the Navier-Stokes equations on unstructured grids. All of the computed cases include the main wing with a half-span flap deflected to 39 degrees and a three-quarter-span slat deflected to 6 degrees. Computations of the model, which simulates a landing configuration at 10 degrees angle of attack and a chord-based Reynolds number of 3.7 million, are validated with surface pressure measurements acquired at the NASA Ames 7- by 10-Foot Wind Tunnel. The results increase the computational knowledge of how to accurately model the flow physics of a multi-element wing with three-dimensional flow by using DetachedEddy Simulation.
\end{abstract}

\section{NOMENCLATURE}

$\begin{array}{ll}a & \text { acoustic speed, } \mathrm{ft} / \mathrm{s} \\ b & \text { wing span, } 5 \mathrm{ft} \\ C_{D} & \text { wing drag coefficient, } D /\left(q_{\infty} S\right) \\ C_{L} & \text { wing lift coefficient, } L /\left(q_{\infty} S\right) \\ C_{p} & \text { pressure coefficient, }\left(p-p_{\infty}\right) / q_{\infty} \\ c & \text { wing chord, } 2.5 \mathrm{ft} \\ D & \text { wing drag, lb } \\ f & \text { frequency } \\ k & \text { thermal conductivity } \\ k_{t} & \text { turbulent thermal conductivity } \\ L & \text { wing lift, lb } \\ M & \text { Mach number, } V / a\end{array}$

* Distinguished Visiting Professor, on leave from the Department of Aerospace Engineering, California Polytechnic State University. Associate Fellow AIAA.

** Associate Professor. Associate Fellow AIAA.

$\dagger$ Senior Member AIAA.

This material is declared a work of the U.S. Government and is not subject to copyright protection in the United States.

$\begin{array}{ll}p & \text { static pressure, } \mathrm{lb} / \mathrm{ft}^{2} \\ q & \text { dynamic pressure, } \rho V^{2} / 2 \\ R_{e} & \text { Reynolds number, } \rho V_{c} / \mu \\ S & \text { wing planform area, } 12.5 \mathrm{ft}^{2} \\ S t & \text { Strouhal number, } f c / V_{\infty} \\ t & \text { physical time, sec } \\ t^{*} & \text { computational time, } t V_{\infty} / c \\ V & \text { velocity, } \mathrm{ft} / \mathrm{s} \\ x & \text { streamwise coordinate, } \mathrm{ft} \\ y & \text { spanwise coordinate, } \mathrm{ft} \\ \alpha & \text { angle of attack, deg } \\ \mu & \text { viscosity } \\ \mu_{t} & \text { turbulent eddy viscosity } \\ \rho & \text { density, slug/ft }{ }^{3} \\ \infty & \text { freestream condition }\end{array}$

INTRODUCTION

One current challenge for Computational Fluid Dynamics (CFD) research is the accurate prediction of high-lift flows. Aircraft in cruise configurations usually have wings consisting of a single, simply-connected geometry. However, typical high-lift systems for both transports and military aircraft employ both leading-edge devices (such as slats) and trailing-edge devices (such as slotted flaps) which are separated from the main wing element by very small gaps. For realistic three-dimensional configurations, these devices extend only partially across the span of the wing and lie in close proximity to structural elements such as supporting brackets, engines, and pylons.

The high-lift system also introduces complex flow physics. In his classic 1974 lecture, A.M.O. Smith describes the effects of the lifting performance of multiple-element airfoils with properly designed gaps. ${ }^{1}$ Many of these concepts can be illustrated using potential flow relationships between the various elements of the high-lift airfoil. Nevertheless, high-lift 
system performance is dominated by viscous effects. Meredith ${ }^{2}$ enumerates some of the viscous phenomena for multi-element airfoils:

1. boundary layer transition

2. shock and boundary layer interaction

3. viscous wake interactions

4. confluent wakes and boundary layers

5. flow separation

Each of these viscous flow phenomena pose extreme difficulties for CFD modeling.

Many two-dimensional viscous NavierStokes simulations of the flow over multi element airfoils have been reported. These studies range from the early work by Shuster and Birckelbaw ${ }^{3}$ to studies by Rumsey et al. ${ }^{4}$ and Fejtek. ${ }^{5}$ Rumsey et al. investigated the flow over two different multielement airfoils. They concluded that specification of transition location is crucial to the accurate computation of boundary layer profiles, that the prediction of the slat flowfield is difficult, and that the performance of eddy viscosity turbulence models and a nonlinear explicit algebraic stress model are similar for this flow. Fejtek ${ }^{5}$ surveyed the results of a code validation challenge sponsored by the CFD Society of Canada; the test case was a three-element airfoil configured for take-off.

Due to geometric and physical complexities, as well as the associated intense resource requirements for high-lift CFD, reports on threedimensional Navier-Stokes applications are limited. In 1993, Rogers ${ }^{6}$ demonstrated overset grid techniques for the main wing and flap elements of a T-39 Sabreliner aircraft, although the grid density was too coarse to properly resolve the flowfield. The following year, Mathias et al. ${ }^{7-8}$ computed the flow about a twoelement, unswept wing with a part-span flap using an incompressible Navier-Stokes code. The computations captured the flow near the flap-edge and compared favorably with experimental data. From 1995 to the present, similar vis cous computations for unswept, twoelement wings with part-span flaps have been reported by Jones et al. ${ }^{9}$ who also used an overset approach, by Khorrami et al. ${ }^{10-11}$ who applied a patched grid approach and focused on the airframe noise aspects of the flow, and by Mavriplis and Venkatakrishnan ${ }^{12}$ and Anderson et al. ${ }^{13}$ who each investigated unstructured grid approaches. Recent investigations have addressed more realistic high-lift geometries. For example, Rogers, $\mathrm{CaO}$, and $\mathrm{Su}^{14}$ applied and evaluated the overset grid method for a Boeing
747PD high-lift configuration. Also, Baker, et $\mathrm{al}^{15}$ have applied the overset grid method for a part-span slat/part-span flap configuration using the Spalart-Almaras turbulence model.

A good review of computational capabilities for simulating high-lift wings was recently conducted by Rumsey and Ying. ${ }^{16}$ They concluded that there were three main areas of improvement required for the prediction of highlift system aerodynamics:

1. including unsteady effects

2. improving turbulence models

3. improving modeling fidelity

This study extends the previous threedimensional, high-lift CFD research in two of the three ways suggested by Rumsey and Ying: by adding unsteady effects to the computations, and by using a higher-order hybrid RANS/LES turbulence model. This article contains a description of the high-lift wing geometries with part-span slat and flap elements. A brief description of the Cobalt Navier-Stokes algorithm is provided, as well as descriptions of the Spalart-Allmaras turbulence model and Detached-Eddy Simulation concept. Results are presented and compared with available wind tunnel data.

\section{HIGH-LIFT WING GEOMETRY}

The baseline wing for this investigation consists of a NACA $63_{2}-215$ Mod. B airfoil section. $^{17}$ Figure 1 shows the rigging of the complete three-element section, and Fig. 2 shows the full wing geometry. The reference chord length, $c$, for the unflapped section of the wing is $2.5 \mathrm{ft}$. Across one-half of the span, a 30\% chord Fowler flap was installed. The flap was deflected 39 degrees with a gap of $2.7 \%$ chord and an overlap of $1.5 \%$ chord. The part-span slat was deflected 6 degrees and deployed to a gap of $2.0 \%$ chord and an overlap of $-0.5 \%$ chord.

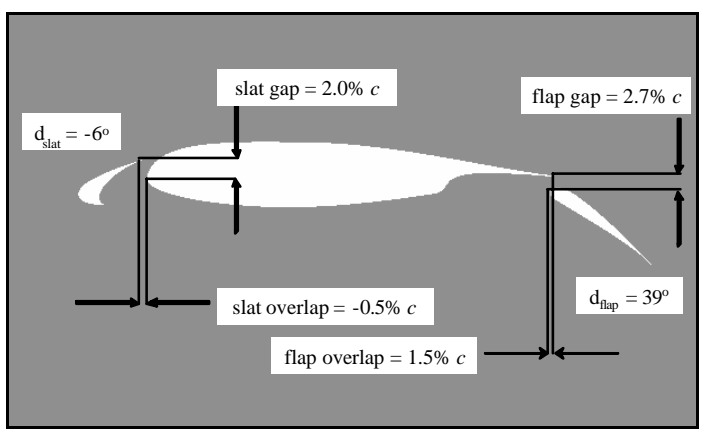

Figure 1. NACA 632-215 Mod. B airfoil section with slat and Fowler flap. 


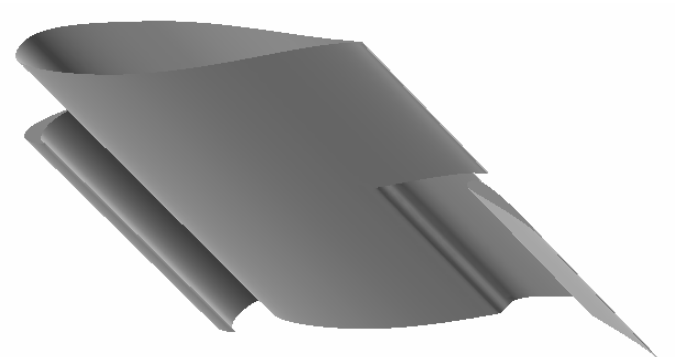

Figure 2. Part-span flap/part-span slat wing geometry.

These configurations were experimentally studied in a series of tests conducted in the NASA Ames 7-by-10-Foot Wind Tunnel by Storms et al. ${ }^{18}$ The model vertically spanned the tunnel between two splitter-plate walls thereby reducing the effective test section size to $5 \mathrm{ft}$. by $10 \mathrm{ft}$., or 2 chords by 4 chords. The experimental repeatability of total lift coefficient was \pm 0.01 for an actual $C_{L}$ less than or equal to $0.95 C_{L_{M A X}}$. The total lift coefficient was based upon integration of pressure measurements. The pressure measurements were made with pressure transducers having different ranges: $0.31 \%$ for $C_{p}>-1,0.28 \%$ for $-1>C_{p}>-2,0.26 \%$ for $-2>$ $C_{p}>-5$, and $0.21 \%$ for $C_{p}<-5$. Since the majority of pressure coefficients measured were between $C_{p}=1$ and $C_{p}=-3$, the average uncertainty is approximately $0.30 \%$.

Figure 3 shows a planform view of the model and some of the spanwise locations where surface pressure measurements were acquired. To quantify the influence of a slat, three different leading-edge configurations were investigated: a two-element wing without a slat, a three-element wing with a full-span slat, and a three-element wing with a three-quarter-span slat. Trip discs were placed at $0.05 c$ and $0.10 c$ on the upper and lower surfaces of the main element, respectively, for the two element wing without a slat. No trip discs were used on the three element configurations in the experimental tests.

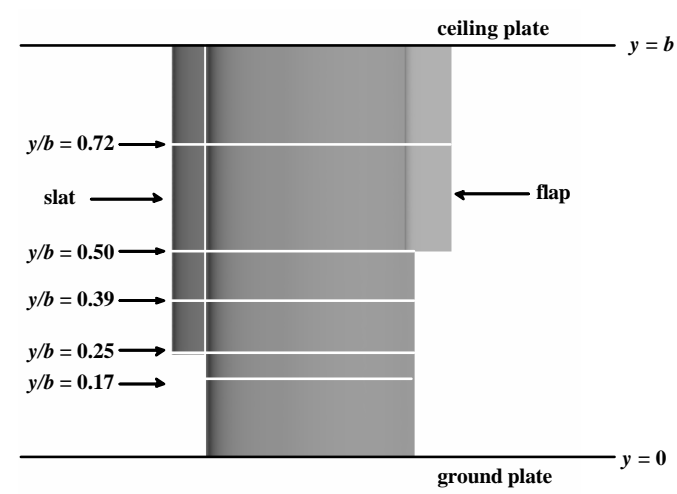

Figure 3. Part-s pan slat/part-span flap wing $(y / b$ locations are noted for pressure tap locations that are considered in this study ).

\section{NUMERICAL APPROACH}

All of the computations were performed using the experimental coordinate system with the wing positioned at 10 degrees angle of attack at a Mach number of 0.22 and a chord-based Reynolds number of $R_{e}=3.7 \times 10^{6}$. The tunnel was modeled according to the experimental test section dimensions ( 2 chords $\times 4$ chords), with the walls being represented with a slip boundary condition. The inlet and outlet planes of the tunnel were placed 10 chords upstream and downstream of the model and were represented with a modified Riemann invariant boundary condition. The multi-element wing was represented with a no-slip boundary condition appropriate for viscous calculations.

\section{Flow Solver and Turbulence Models}

Flow Solver In this section a brief description of the numerical method is provided. Full details of the computational scheme are presented in Ref. 19. Solutions for all configurations were computed with the commercial version of Cobalt developed by Cobalt Solutions, LLC. Cobalt solves the unsteady, three-dimensional, compressible Navier-Stokes equations on a hybrid unstructured grid. The code has several choices of turbulence models, including Spalart Almaras (SA), and Menter's Shear Stress Transport (SST) RANS, as well as DES versions of SA and SST. All simulations were computed on unstructured meshes with prisms in the boundary layer and tetrahedra elsewhere. The computational meshes were generated with the software packages GridTool ${ }^{20}$ and VGRIDns. ${ }^{21}$ 
Turbulence Models For simulation of turbulent flows, the governing equations are suitably averaged, yielding turbulent stresses that require a model. A Boussinesq approximation is invoked in the momentum equations and the turbulent eddy viscosity $\left(\mu_{t}\right)$ is used to relate the stresses to the strain rate. The turbulent heat flux is also modeled using a gradient-transport_hypothesis, requiring specification of a turbulent thermal conductivity, $k_{t}$. The Reynolds analogy is applied and the turbulent heat flux is modeled using a constant turbulent Prandtl number of 0.9 . Using turbulent eddy viscosity and turbulent conductivity, the variable, $\mu$, is replaced by $\left(\mu+\mu_{t}\right)$ and $k$ is replaced by $\left(k+k_{t}\right)$ in the governing equations.

Spalart-Allmaras Turbulence Model The Spalart-Allmaras $^{22}$ one equation model (SA) solves a single partial differential equation for a working variable $\tilde{v}$ which is related to the turbulent viscosity. The differential equation is derived by "using empiricism and arguments of dimensional analysis, Galilean invariance and selected dependence on the molecular viscosity." 22 The model includes a wall destruction term that reduces the turbulent viscosity in the laminar sublayer. The model takes the form,

$$
\begin{aligned}
& \frac{D \tilde{v}}{D t}=c_{b 1} \tilde{S} \tilde{v}-c_{w 1} f_{w}\left[\frac{\tilde{v}}{d}\right]^{2} \\
& +\frac{1}{\sigma}\left[\nabla \cdot((v+\tilde{v}) \nabla \tilde{v})+c_{b 2}(\nabla \tilde{v})^{2}\right]
\end{aligned}
$$

The turbulent kinematic viscosity is obtained from,

$$
v_{t}=\frac{\mu_{t}}{\rho}=\tilde{v} f_{v 1} ; f_{v 1}=\frac{\chi^{3}}{\chi^{3}+c_{v 1}^{3}} ; \chi=\frac{\tilde{v}}{v}
$$

where $S$ is the magnitude of the vorticity given by

$$
S=|\omega|=|\nabla \times(u \hat{i}+v \hat{j}+w \hat{k})|
$$

and the modified vorticity is,

$$
\tilde{S}=S+\frac{\tilde{v}}{\kappa^{2} d^{2}} f_{v 2} ; f_{v 2}=1-\frac{\chi}{1+\chi f_{v 1}}
$$

where $d$ is the distance to the closest wall. The wall destruction function $f_{w}$ is,

$$
f_{w}=g\left[\frac{1+c_{w 3}^{6}}{g^{6}+c_{w 3}^{6}}\right]^{\frac{1}{6}}
$$

and

$$
g=r+c_{w 2}\left(r^{6}-r\right) ; r \equiv \frac{\tilde{v}}{\tilde{S} \kappa^{2} d^{2}}
$$

The turbulent viscosity is obtained from the turbulent kinematic viscosity by $\mu_{t}=\rho v_{t}$. The model coefficients are given in Table 1.

\begin{tabular}{|c|c|c|}
\hline$c_{b 1}=0.1355$ & $c_{b 2}=0.622$ & $c_{v 1}=7.1$ \\
\hline$\kappa=0.41$ & & $\sigma=2 / 3$ \\
\hline $\begin{array}{c}c_{w 1}=c_{b 1} / \kappa^{2} \\
+\left(1+c_{b 2}\right) / \sigma\end{array}$ & $c_{w 2}=0.3$ & $c_{w 3}=2$ \\
\hline
\end{tabular}

Table 1. Spalart-Allmaras turbulence model coefficients.

Detached-Eddy Simulation The DetachedEddy Simulation method was proposed by Spalart et al. ${ }^{23}$ and was originally based on the Spalart-Allmaras one equation RANS turbulence model (detailed above) with a more detailed presentation in Ref. 22. The wall destruction term presented above is proportional to $(\tilde{v} / d)^{2}$, where $d$ is the distance to the wall. When this term is balanced with the production term, the eddy viscosity becomes proportional to $\hat{S} d^{2}$, where $\hat{S}$ is the local strain rate. The Smagorinski LES model varies its sub-grid scale (SGS) turbulent viscosity with the local strain rate, and the grid spacing: $v_{S G S} \propto \hat{S} \Delta^{2}$, where $\Delta=\max (\Delta x, \Delta y, \Delta z)$. If $d$ is replaced with $\Delta$ in the wall destruction term, the SA model will act as a Smagorinski LES model.

To exhibit both RANS and LES behavior, $d$ in the SA model is replaced by

$$
\tilde{d}=\min \left(d, c_{D E S} \Delta\right)
$$

When $d<<$, the model acts in a RANS mode and when $d \gg \Delta$ the model acts in a Smagorinski LES mode. Therefore the model switches into LES mode when the grid is locally refined.

DES was implemented in an unstructured grid method by Forsythe et al. ${ }^{24}$ They determined that the DES constant should be $c_{D E S}=0.65$, consistent with the structured grid implementation of Spalart et al. ${ }^{23}$ when the grid spacing, $\Delta$, was taken to be the longest distance 
between the cell center and all of the neighboring cell centers.

A Newton sub-iteration method is used in the solution of the system of equations to improve time accuracy of the point implicit method and approximate Jacobians. In the calculations presented below, a typical number of three Newton sub-iterations is used for all time-accurate cases.

Grid Generation Spalart ${ }^{25}$ described the process of grid design and assessment for DES, defining important regions of the solution and offering guidelines for grid densities within each region. The "Young-Person's Guide" (YPG) ${ }^{25}$ forms a basis for interpretation of many of the esults presented below. One of the traditional motivations for using unstructured grids has been the ability to rapidly create grids around complex geometries. There are other positive attributes of unstructured grids that are relevant to DES. Most notably, it is possible to concentrate points in the region of interest (i.e. the vortex core or aft of breakdown) and rapidly coarsen the grid away from these areas. This region of interest was termed the "focus region" in the YPG.

Another advantage exploited in the present study is the isotropic cells generated in the LES region by most unstructured grid generation packages. The YPG reference describes the desirability of having nearly isotropic grid cells in the focus region in which unsteady, timedependent, features are resolved. For this reason, unstructured grids are good candidates for use in DES because near isotropy of the grid cells in the LES region is assured by most grid generation packages.

Morton et al. ${ }^{26}$ applied the YPG guidelines to three massively separated flows of interest: forebody in a cross-flow, flow over a delta wing at $27^{\circ}$ angle of attack, and the flow over an F$15 \mathrm{E}$ at $65^{\circ}$ angle of attack. In the latter two cases an extensive grid sensitivity study was performed by systematically varying the grid by a scale parameter allowing a very consistent analysis of grid effects when using the DES method of computing massively separated flows. DES of the F-15E provided an impressive drag coefficient match of $5 \%$ to the Boeing flight test data at $65^{\circ}$ angle of attack. A more detailed look at the simulations can be found in Ref. 27.

Another important grid technology that is particularly well suited for DES is adaptive mesh refinement. Pirzadeh ${ }^{28}$ presented a method based on a tetrahedral unstructured grid technology developed at NASA Langley Research Center with application to two configurations with vortex dominated flowfields. The large improvement of the adapted solutions in capturing vortex flow structures over the conventional unadapted results was demonstrated by comparisons with wind tunnel data. Pirzadeh showed the numerical prediction of these vortical flows was highly sensitive to the local grid resolution and he also stated that grid adaptation is essential to the application of CFD to these complicated flowfields. His most successful computations were performed using an inviscid method due to the inadequacies of standard turbulence models in computing these complicated flowfields. For the current work a mean flow solution on a baseline grid is used to create an adaptively refined mesh and the new grid is used with DES to compute the unsteady flowfield.

The GridTool $^{20}$ and VGRIDns ${ }^{21}$ grid generation methods were applied to the surface geometry for the part-span slat/part-span flap configuration; representative grids show the clustering near the leading and trailing edges, as well as the grid density elsewhere around the wing (see Figs. 4 and 5). When this geometry was modeled previously, ${ }^{15}$ the Chimera overset grid proces ${ }^{29,30}$ was used due to the combination of one-, two-, and three-element airfoil sections that abut one another. The use of unstructured grids has greatly reduced the amount of time required for grid generation, and should show itself to be a valuable tool for this type of configuration.

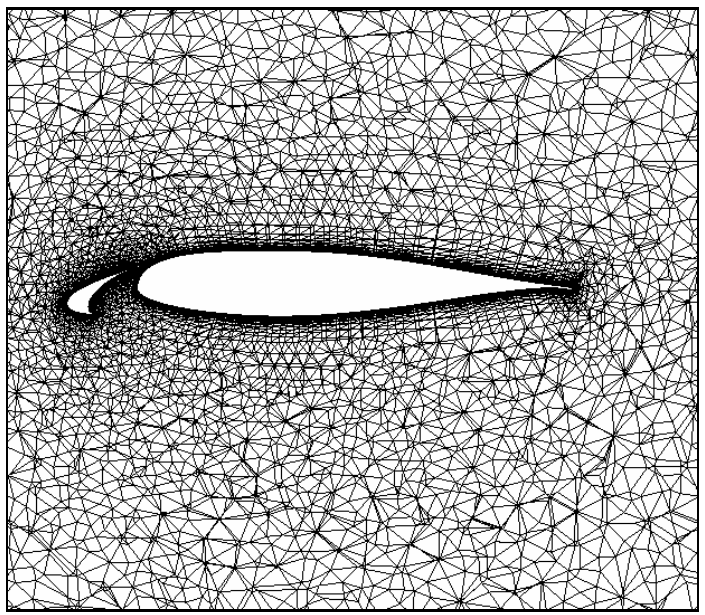

Figure 4. Spanwise cut of unstructured grid for wing at $y / b=0.25$. 


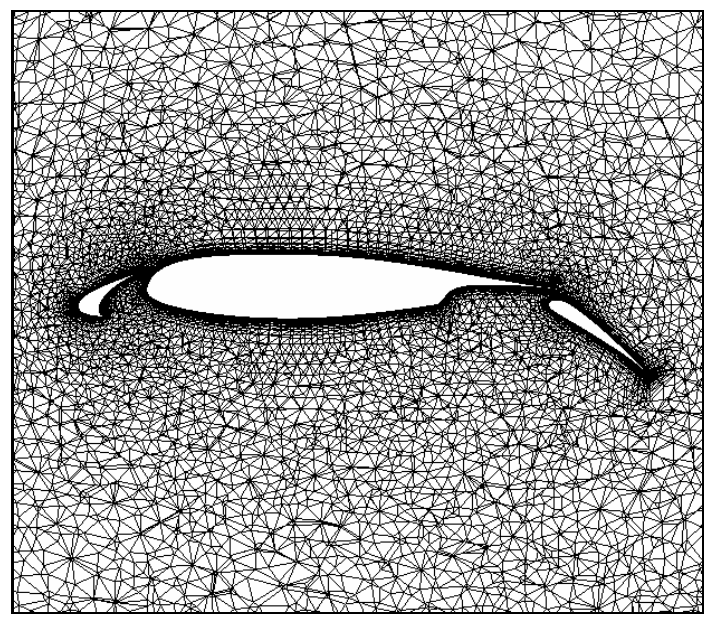

Figure 5. Spanwise cut of unstructured grid for wing at $y / b=0.75$.

Four initial grids were created of 2.4 million, 27 million, 3.2 million, and 5.6 million cells each. These grids were created using global refinement, so that all areas of the grid received additional grid support, whether it was required or not. A grid sensitivity study was performed for the full configuration with all four grids to determine the grid fineness required to reach grid independence. Figure 6 shows the time convergence histories for the four grids, with some unsettling results being seen. While all four grids achieved approximately similar lift results, there was no apparent convergence to a single result, and as can be seen with the 5.6 million cell grid, evidence of unsteadiness became apparent as the grid was refined. It should be noted that without a priori knowledge of the wind tunnel results, it would have been easy to assume that the 3.2 million cell grid was acceptable, since it was showing signs of having achieved similar results to the smaller grids. However, all of the grids discussed predicted that the flow over the flapped element was largely stalled, while the wind tunnel data shows the flow over most of the the flap to be attached for approximately $80 \%$ of the flap chord. This difference is most likely due to a lack of grid density in the cove and flap gap regions, although further grid refinement did not predict attached flow over the flap. It is also interesting that the three smallest grids first converged to $C_{L}$ $=0.25$ after about 500 iterations, and then reconverged to $C_{L}=0.21$. At 500 iterations the computations showed the flow over the flap to be attached, but after 500 iterations the flap flow became separated and would not reattach.

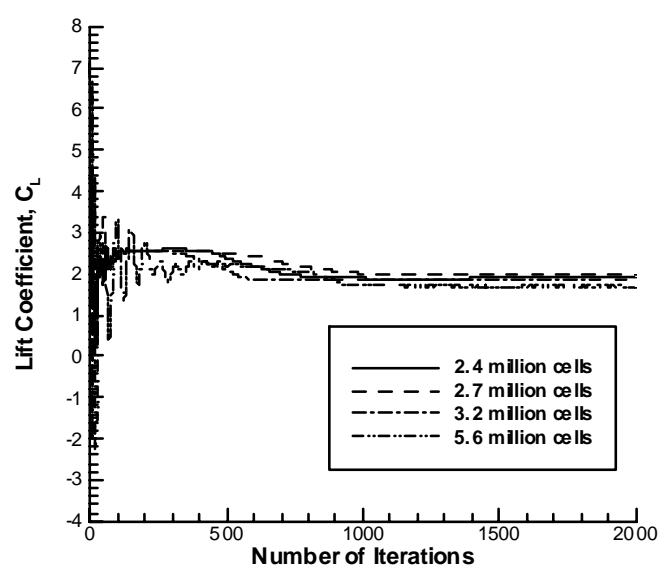

Figure 6. Convergence history for four initial grids.

Adaptive mesh refinement $(\mathrm{AMR})^{28}$ was used to refine the mesh at various locations in the flowfield, including the slat, main element leading edge, and cove/flap regions. The refined mesh contained 5.2 million cells, which was approximately the same as the largest grid used earlier since the mesh size in the farfield was reduced in order to maintain a reasonable grid size.

The AMR grid was run in time accurate mode (the results in Fig. 6 were all run as steady calculations), and the convergence history is presented in Fig. 7, where it is readily apparent that the flowfield is unsteady. While further grids and time steps should be run to consider the flow prediction to be grid and time-step independent, all further results will be presented using this grid.

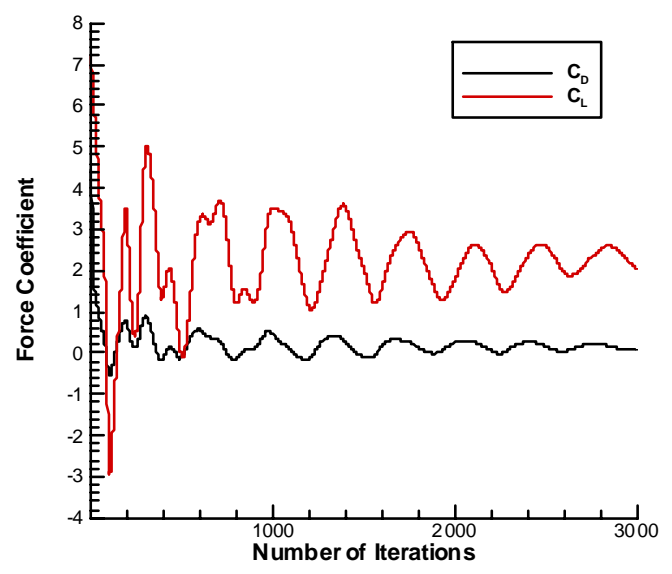

Figure 7. Convergence history for grid created with adaptive mesh refinement. 


\section{SLAT AND FLAP AERODYNAMICS}

\section{Experimental Verification}

Surface pressure coefficient distributions from the wind tunnel test ${ }^{18}$ are shown in Fig. 8. Pressure coefficient distributions are shown at $y / b=0.17$ (unflapped section), $y / b=0.39$ (slat and main element section), $y / b=0.53$ (slat, flap, and main element section near the flap tip), and $y / b=0.72$ (slat, flap, and main element section). The flow over the single element section (no slat or flap) is shown in Fig. 8a, and a representative section between the beginning of the slat and flap is shown in Fig. 8b. These two sections seem relatively normal for single and twoelement airfoils. The most interesting result appears in Fig. 8c, where it is evident that the flap is stalled over most of the upper surface, but further inboard on the flap the flow is attached, as shown in Fig. 8d.

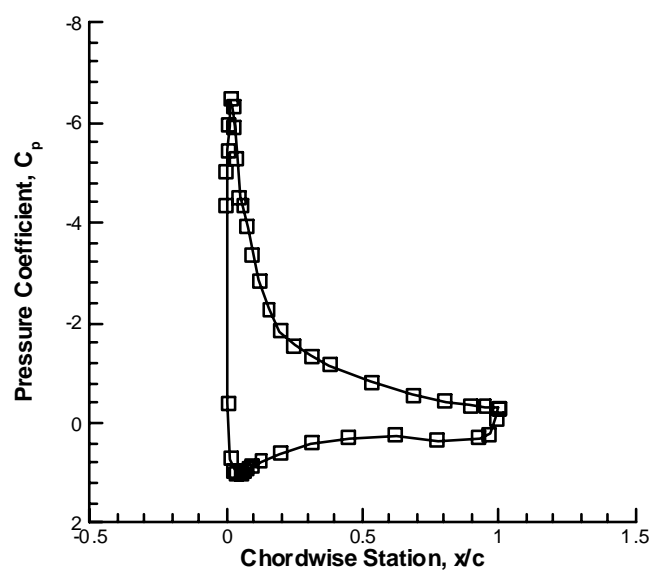

a) $y / b=0.17$

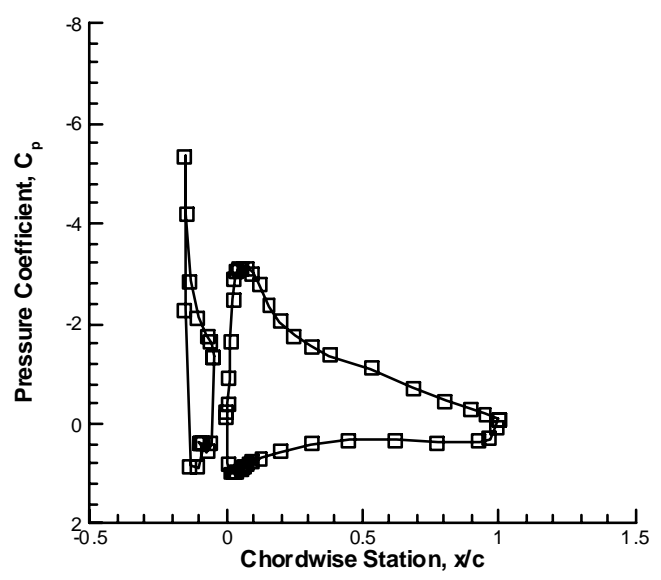

b) $y / b=0.39$

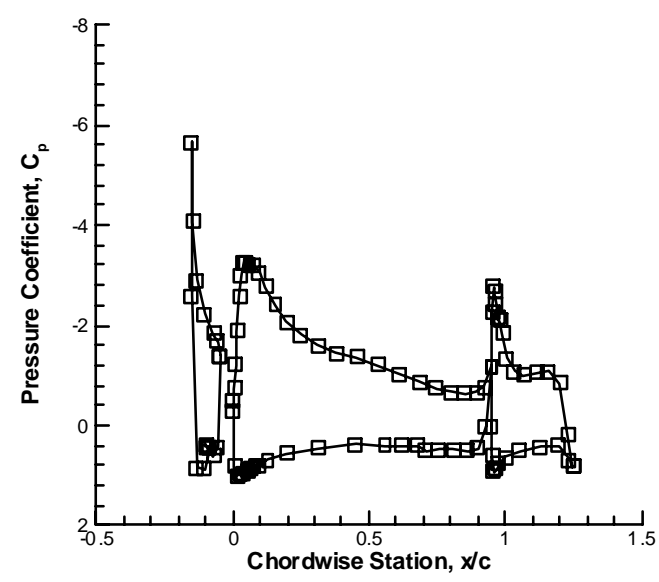

c) $\mathbf{y} / \mathrm{b}=\mathbf{0 . 5 3}$

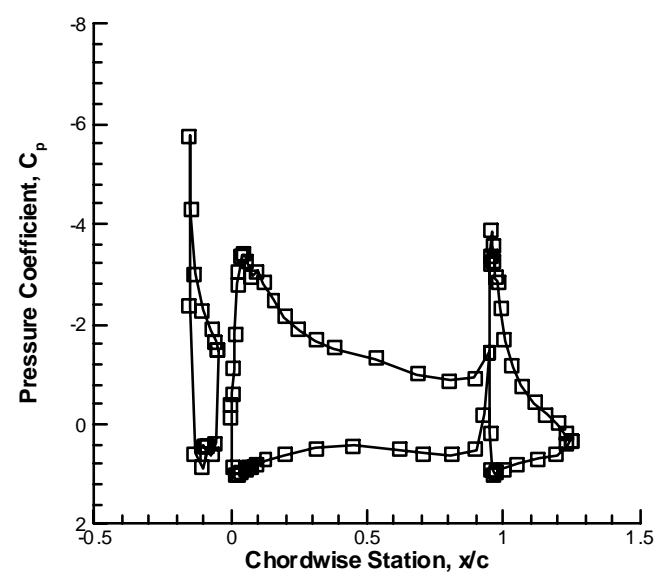

d) $y / b=0.72$

Figure 8. Experimental surface pressures at four spanwise locations.

Computed pressure coefficient distributions at $y / b=0.17$ (unflapped section), $y / b=0.39$ (slat and main element section), and $y / b=0.72$ (slat, flap, and main element section) are shown in Fig. 9 for the AMR grid. The pressure distribution for $y / b=0.17$ (Fig. 9a) is fairly well predicted, with the leading edge suction being under-predicted. This type of under-prediction is usually overcome through using a finer grid density in the leading-edge region of the airfoil, and probably does not affect the overall results of the study. Likewise, the flow over the slat/main element section $(y / b=0.39)$ is fairly well predicted (see Fig. 9b), with only the slat leading-edge suction being under-predicted, although the main element leading-edge suction is well predicted. 


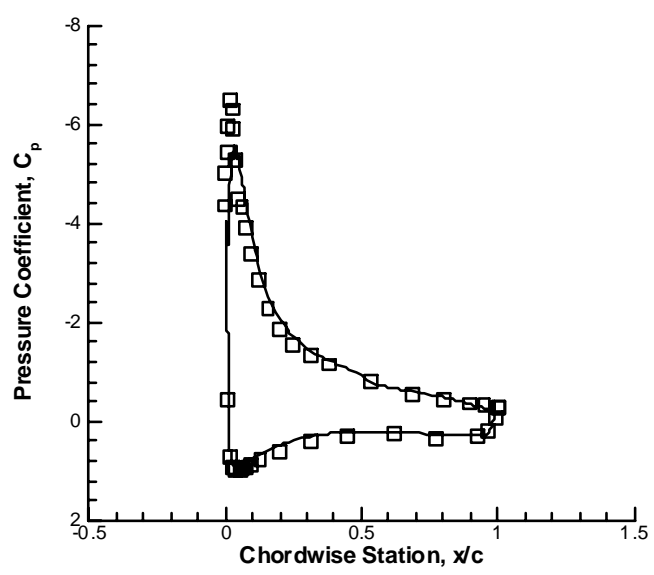

a) $y / b=0.17$

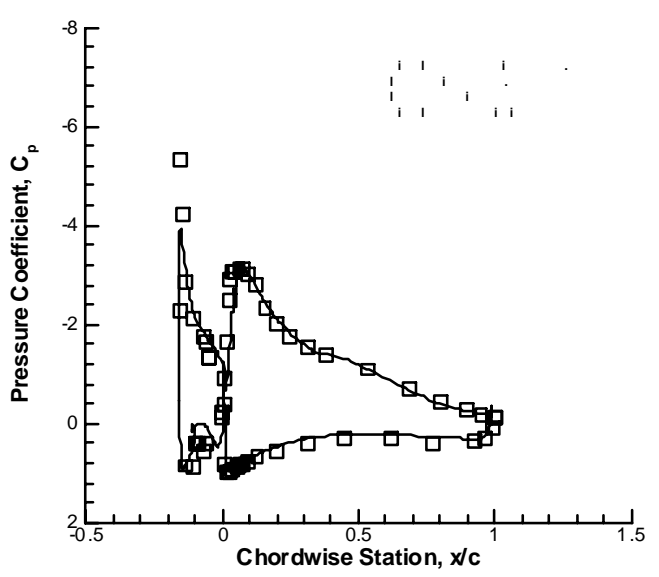

b) $y / b=0.39$

The most disappointing results appear in Fig. 9c $(y / b=0.72)$, where the flap was computed with separated flow, but the wind tunnel data shows the flow to be attached. The prediction of the upper surface pressures for the flap seem much closer to the experimental data for $y / b=0.53$ (see Fig. 8c), where the flow is clearly separated. These results are harder to improve than the under-prediction of leadingedge suction, since flow separation is a function of the flow through the cove region of the flap and through the gap between the flap and the main element. Further studies will address this discrepancy, but the purpose of this work is still in place, since the goal is to see what additional information may be gained by performing calculations with detached-eddy simulation as opposed to just using a RANS model such as the Spalart-Allmaras turbulence model.

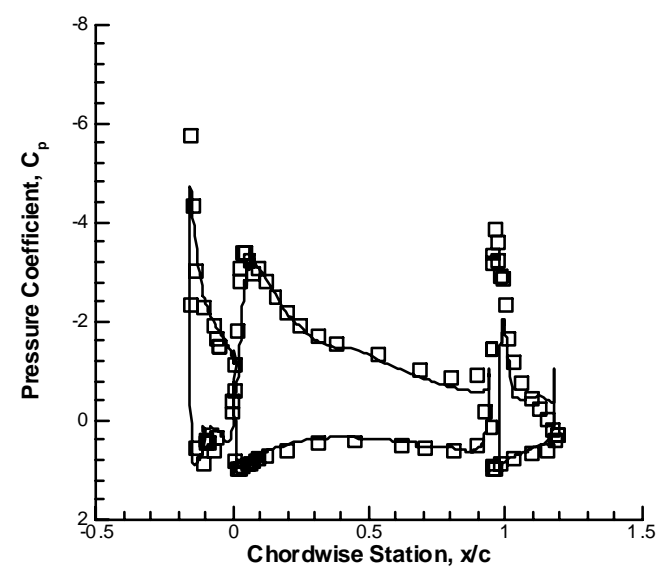

c) $y / b=0.72$

\section{Figure 9. Comparison of computed surface pressures and experimental data at three spanwise locations (? experimental data, ? numerical prediction).}

\section{Physical Explanation of the Flowfield}

Figures 10 through 12 show the upper surface flowfield in three ways: an oil flow simulation, a surface pressure color map, and a vortex particle trace. The oil flow simulation in Fig. 10 helps to show the lines of separation and regions of attached flow on all three elements of the wing. The flow over the slat is clearly attached and aligned with the freestream, a flow condition that continues over the main element behind the slat. In fact, there are no threedimensional effects on the main element behind the slat until the final $30 \%$ of the chord, where the streamlines begin to curve toward the flap. This flow curvature is induced by the flap-tip vortex, which lies below the surface of the main element and induces flow toward the flap on the upper surface of the wing.

The velocity field from the flap-tip vortex is counteracted by the flow induced by the slat-tip vortex, which lies above the surface of the wing. The slat-tip vortex therefore induces flow away from the flap-the closer the streamlines are to the slat-tip vortex, the more they curve away from the flap. Finally, as the streamlines closest to the bottom of the wing are influenced by the slat-tip vortex, an upwash field is induced that causes the flow to separate (see the vertical node near the outboard section of the wing). As was noted in the surface pressure coefficients, the flow over the flap separates at approximately $20 \%$ of the flow chord. 


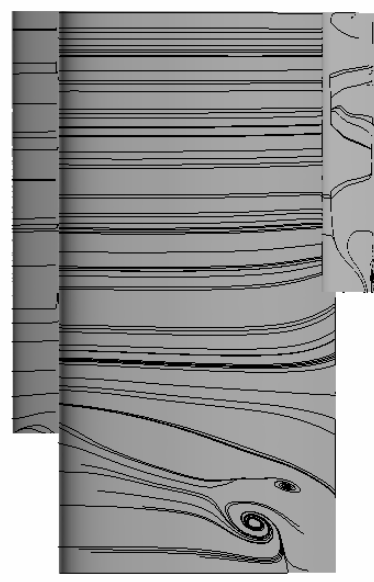

Figure 10. Surface oil flow simulation for part-span flap configuration.

Figure 11 shows the surface pressures using red for high pressure and blue for low pressure. The pressure field shows similar results as mentioned above, however in a different way. The suction peak along the leading edge of the slat and main elements is evident, and the nearly two-dimensional nature of the flow on the main element behind the slat is also obvious. The higher pressures on the aft section of the main element behind the slat-tip vortex are also visible, including the region of separated flow. Finally, the separated flow over the flap is also evident, and the flow over the flap is essentially two-dimensional, except near the flap-tip, where the impact of the flap-tip vortex becomes apparent.

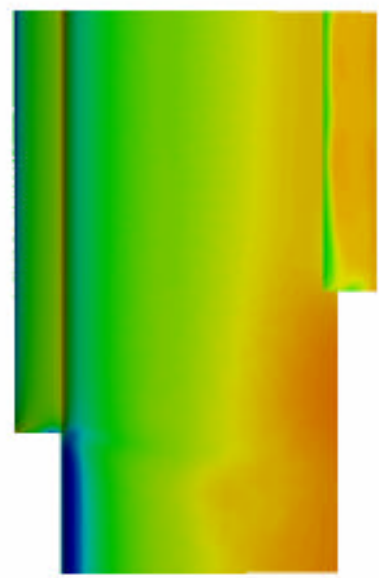

Figure 11. Surface pressures for part-span flap configuration.

Finally, Fig. 12 shows the tracks of the slattip and flap-tip vortices, which help to explain the results seen in Figs. 10 and 11. The slat-tip vortex induces upwash outboard of the slat, which causes the flow to separate on the main element of the wing. The separated flow region, coupled with the influence of the flap-tip vortex, causes the slat-tip vortex to move outboard over the wing, but then to move toward the flap-tip vortex downstream of the wing trailing edge.

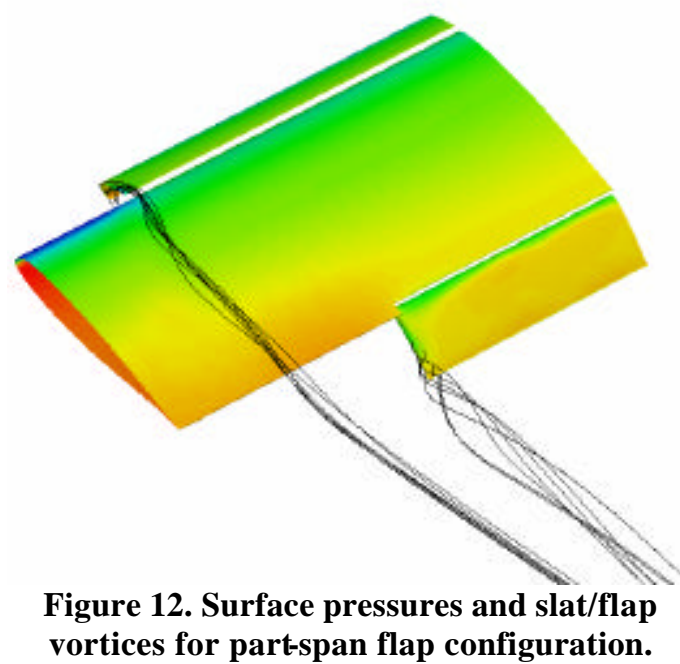

\section{DETACHED-EDDY SIMULATION RESULTS}

The detached-eddy simulation (DES) runs used the mesh created with adaptive mesh refinement, which had 5.21 million cells. Since DES, by its very nature, represents the unsteady flowfield, care must be taken to numerically integrate in a time-accurate sense. Previous calculations of wake-like flowfields by the third author have found that the flow is well modeled using a non-dimensional time step of ? $t^{*}=0.01$. For the freestream velocity of $250 \mathrm{ft} / \mathrm{sec}$ and the chord of $2.5 \mathrm{ft}$, the physical time -step appropriate for this case is approximately $? t=0.0001$. Also, the DES runs were made using three Newton sub-iterations to insure second-order time integration. Also, since it was evident from the previous time-accurate run (see Fig. 7) that the start-up oscillations last for somewhere between 1000 and 2000 iterations, the DES runs were made for 6000 iterations. Results will be shown both for various instances of time and for timeaveraged results for all solution after 3000 iterations.

Figure 13 details the time convergence history for the DES run, which shows that the flow is definitely unsteady with an average lift coefficient of a little over $C_{L}=0.2$, which compares well with the results of Ref. 15. 


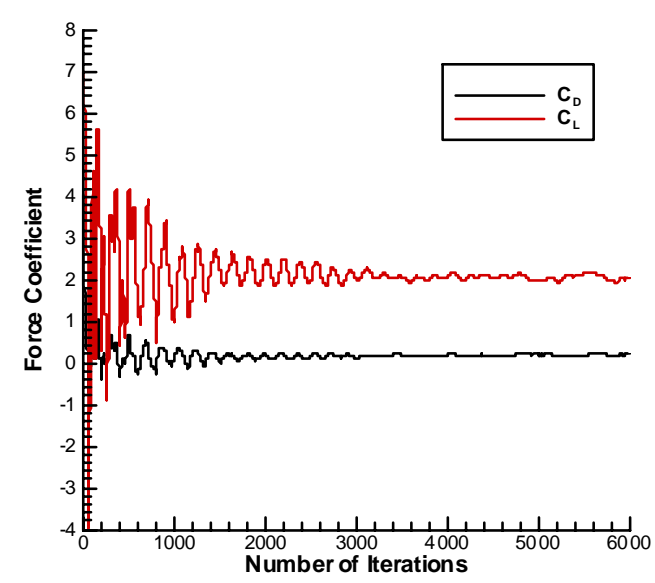

Figure 13. Convergence history for DES run.

The unsteady nature of the flow is primarily caused by the separated flow region downstream of the slat-tip vortex, with the slat-tip vortex position and coherence greatly altered as a function of time. Figure 14 shows the upper surface flowfield of the wing at four times (all results are shown after the flowfield had reached a stationary mean result, as seen in Fig. 13). The flow is visualized by isosurfaces of x-vorticity which have been colored by local static pressure. While the slat-tip vortex is clearly visible in each picture, the size and strength of the vortex changes at each moment in time (compare Figs. $14 \mathrm{a}, 14 \mathrm{~b}, 14 \mathrm{c}$, and 14d).

The vortex appears to breakdown as it flows over the wing, and the induced velocities from the vortex cause flow separation (as was mentioned earlier). The position of flow separation and the size of the separated flow region vary greatly in time. The flap-tip vortex, however, appears to be very steady, with only slight changes in the flowfield over the flap being visible.

During the steady calculations it appeared that the slat-tip and flap-tip vortices were strongly interacting, especially as the slat-tip vortex convected downstream. It is clear in the DES computations that the slat-tip vortex has lost coherence long before it reaches the vicinity of the flap-tip vortex. None of these features was apparent in the steady RANS calculation with the exception of a separated flow region outboard of the slat-tip vortex. The size and shape of that separated flow region changed a great deal once the time-accurate DES solutions were obtained. However, a flow visualization of the slat-tip vortex in the wind tunnel test led to the following observation, "As it is convected further downstream, the slat-tip vortex dissipates due to interaction with the main-element boundary layer. At approximately mid-chord of the main element, the slat-tip vortex is no longer discernable using the smoke flow-visualization technique." 18 It is entirely possible that the experimentalists were seeing a vortex that was breaking down as it reached the stalled region of the wing.

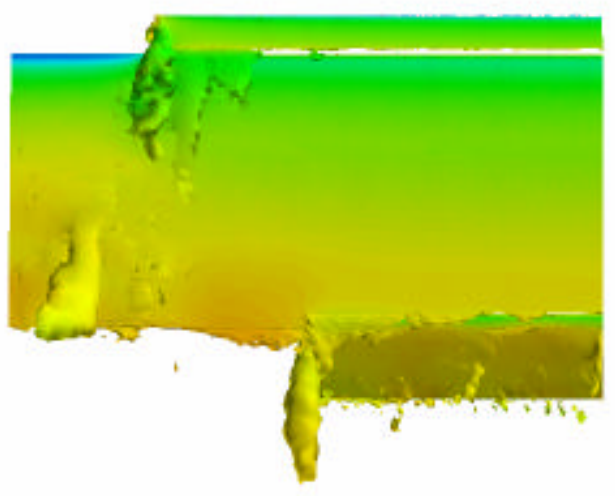

a) 3000 iterations

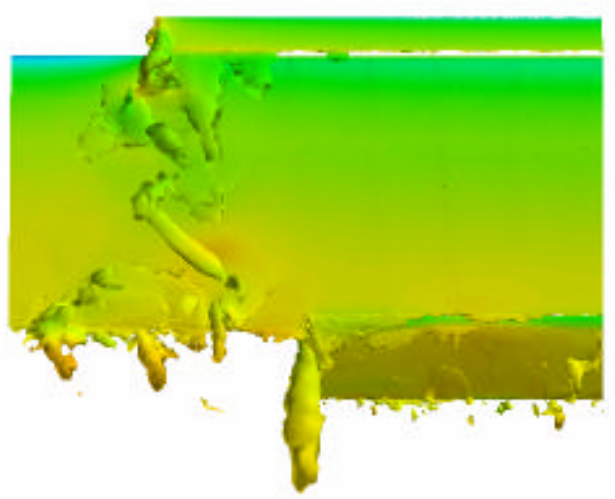

b) 4000 iterations

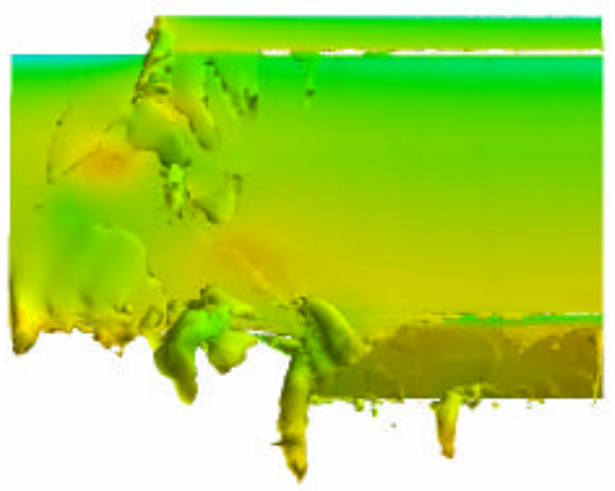

c) 5000 iterations 


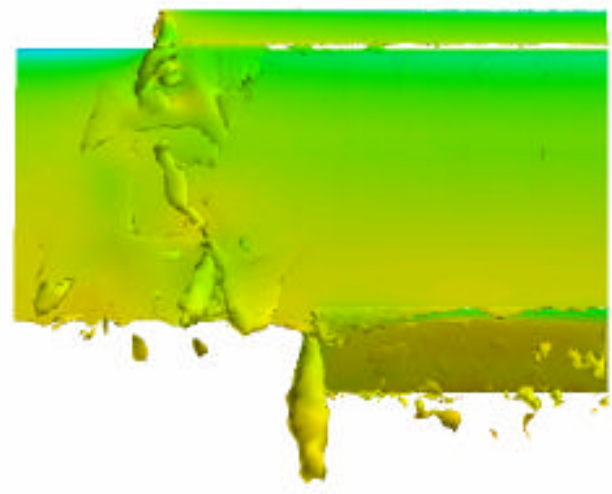

d) 6000 iterations

Figure 14. DES flowfield prediction; $\mathrm{x}$-vorticity isosurfaces colored by pressure.

A power spectrum density of the wing lift was performed and is shown in Fig. 15. Power spikes take place at Strouhal numbers of 0.07, $0.13,0.27,0.4$, and 0.53 , which corresponds to wave numbers of $15.6,7.6,3.8,2.5$, and 1.9, respectively. These frequencies correspond well to oscillations of a vortex breakdown region and vortex instabilities as shown in Fig. $15 .^{31}$ Further studies will correlate these frequencies with specific flow features, but based on early analysis and observation most of these frequencies are related to the separated flow region behind the slat-tip vortex.

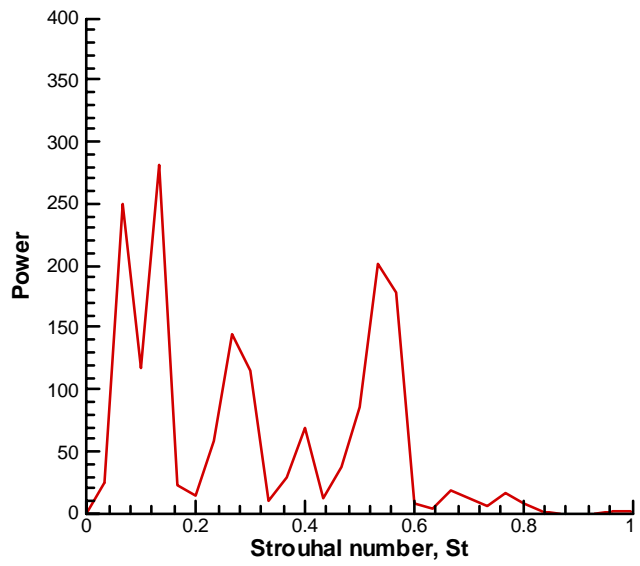

Figure 14. Power spectrum density of wing lift.

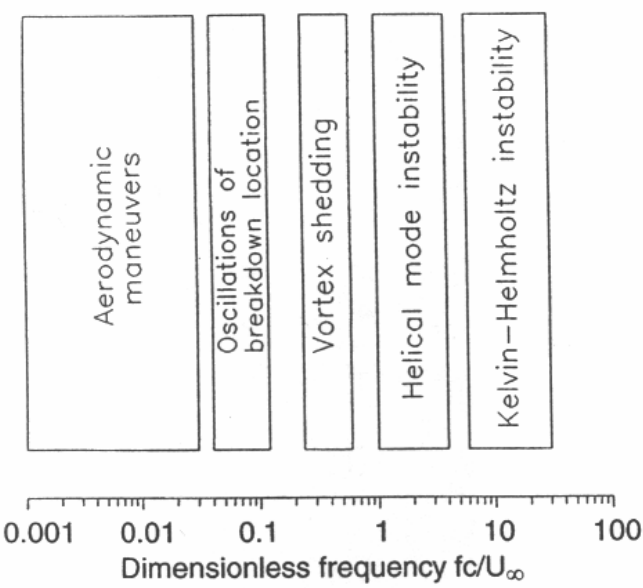

Figure 15. Spectrum of unsteady flow phenomena (Ref. 31).

\section{CONCLUSIONS}

The flow about an unswept, high-lift wing configured with a leading edge device (a threequarter span slat) and a trailing-edge device (a half-span Fowler flap) have been computed using the Cobalt Navier-Stokes solvers on unstructured grids. This study extends previous high-lift CFD research on the unswept high-lift wing by computing the flow using the hybrid RANS/LES Detached-Eddy Simulation method. Results for the wing flowfield have been compared with existing wind tunnel surface pressure measurements. Examination of computed surface pressure distributions and particle traces for the three high-lift wings verify the fundamental impact of the slat element on the high-lift flowfield. Further, knowledge of the three-dimensional influence of the slat and computational techniques for resolving the flow in the vicinity of a slat tip has been gained and will be applied in future simulations. Specifially, the slat-tip vortex has been found to cause a flow separation that is highly unsteady and disruptive of the lift on the wing main element. Future work should improve the grid density and further develop the importance of the unsteady flowfield on the wing aerodynamics.

\section{ACKNOWLEDGEMENTS}

The authors want to thank Bruce Storms and James Ross of NASA Ames Research Center for supplying the wind tunnel data, and Stuart Rogers of NASA Ames Research Center for supplying the surface geometry for the high-lift wing. 


\section{REFERENCES}

1. Smith, A.M.O., "High-Lift Aerodynamics," AIAA Paper 74-0939, Aug. 1974.

2. Meredith, P.T., "Viscous Phenomena Affecting High-Lift Systems and Suggestions for Further CFD Development," AGARD CP-515, Paper No. 19, Oct. 1992.

3. Shuster, D.M. and Birckelbaw, L.D., "Numerical Computation of Viscous Flowfields about Multiple Component Airfoils," AIAA Paper 85-0167, Jan. 1985.

4. Rumsey, C.L., Gatski, T.B., Ying, S.X., and Bertelrud, A., "Prediction of High-Lift Flows using Turbulent Closure Models," AIAA Journal, Vol. 36, No. 5, 1998, pp. 765-774.

5. Fejtek, I., "Summary of Code Validation Results for a Multiple Element Airfoil Test Case," AIAA Paper 97-1932, July 1997.

6. Rogers, S.E., "Progress in High-Lift Aerodynamic Calculations," Journal of Aircraft, Vol. 31, No. 6, 1994, pp. 1244 1251.

7. Mathias, D.L., "Navier-Stokes Analysis of the Flow about a Flap Edge," M.S. Thesis, California Polytechnic State University, San Luis Obispo, CA, June 1994.

8. Mathias, D.L., Roth, K.R., Ross, J.C., Rogers, S.E., and Cummings, R.M., "Navier-Stokes Analysis of the Flow about a Flap-Edge," Journal of Aircraft, Vol. 35, No. 6, 1998, pp. 833-838.

9. Jones, K.M., Biedron, R.T., and Whitlock, M., "Application of a Navier-Stokes Solver to the Analysis of Multielement Airfoils and Wings Using Multizonal Grid Techniques," AIAA Paper 95-1855, June 1995.

10. Khorrami, M.R., Singer, B.A., and Takallu, M.A., "Analysis of Flap Side-Edge Flow Field for Identification and Modeling of Possible Noise Sources," SAE Transactions, Vol. 106, No. 2, 1997, pp. 2716-2722.

11. Khorrami, M.R., Singer, B.A., and Radeztsky, R.H., "Reynolds Averaged Navier-Stokes Computations of a Flap SideEdge Flow Field," AIAA Journal, Vol. 37, No. 1, 1999, pp. 14-22.

12. Mavriplis, D.J. and Venkatakrishnan, V., "A 3-D Agglomeration Multigrid Solver for the Reynolds-Averaged Navier-Stokes Equations on Unstructured Meshes," International Journal for Numerical Methods in Fluids, Vol. 23, No. 6, 1996, pp. 527-544.
13. Anderson, W.K., Rausch, R.D., and Bonhaus, D.L., "Implicit/Multigrid Algorithms for Incompressible Turbulent Flows on Unstructured Grids," Journal of Computational Physics, Vol. 128, 1996, pp. 391-408.

14. Rogers, S.E., Cao, H.V., and Su, T.Y., "Grid Generation for Complex High-Lift

Configurations," AIAA Paper 98-0311, June 1998.

15. Baker, M.D., Mathias, D.L., Roth, K.R., and Cummings, R.M., "Numerical Investigation of Slat and Compressibility Effects for a High-Lift Wing," Journal of Aircraft, Vol. 39, No. 5, 2002, pp. 876-884.

16. Rumsey, C.L and Ying, S.X., "Prediction of High Lift: Review of Present CFD Capability," Progress in Aerospace Sciences, Vol. 38, 2002, pp. 145-180.

17. Hicks, R.M. and Schairer, E.T., "Effects of Upper Surface Modification on the Aerodynamic Characteristics of the NACA 632-215 Airfoil Section," NASA TM 78503, 1979.

18. Storms, B.L., Ross, J.C., Horne, W.C., Hayes, J.A., Dougherty, R.P., Underbrink, J.R., Scharpf, D.F., and Moriarty, P.J., "An Aeroacoustic Study of an Unswept Wing with a Three-Dimensional High-Lift System," NASA TM 112222, Feb. 1998.

19. Strang, W.Z., Tomaro, R.F., Grismer, M.J., "The Defining Methods of Cobalt: A Parallel, Implicit, Unstructured Euler/Navier-Stokes Flow Solver," AIAA Paper 99-0786, Jan. 1999.

20. Samareh, J., "Gridtool: A Surface Modeling and Grid Generation Tool," Proceedings of the Workshop on Surface Modeling, Grid Generation, and Related Issues in CFD Solution, NASA CP-3291, May 9-11, 1995.

21. Pirzadeh, S., "Progress Toward a UserOriented Unstructured Viscous Grid Generator," AIAA Paper 96-0031, Jan. 1996.

22. Spalart, P. R., and Allmaras, S.R., "A One Equation Turbulence Model for Aerodynamic Flows," La Recherche Aerospatiale, Vol. 1, 1994, p. 5.

23. Spalart, P. R., Jou W-H., Strelets M., and Allmaras, S. R., "Comments on the Feasibility of LES for Wings, and on a Hybrid RANS/LES Approach," Advances in DNS/LES, 1st AFOSR Int. Conf. on DNS/LES, Aug 4-8, 1997, Greyden Press, Columbus Oh. 
AIAA PAPER 2004-1233

24. Forsythe, J.R., Ho ffmann, K.A., Dieteker, F.F., "Detached-Eddy Simulation of a Supersonic Axisymmetric Base Flow with an Unstructured Flow Solver," AIAA Paper 2000-2410, June 2000.

25. Spalart, P., "Young-Person's Guide to Detached-Eddy Simulation Grids," NASA CR 2001-211032, July 2001.

26. Morton, S.A., Forsythe, J.R., Squires, K.D., and Wurtzler, K.E., "Assessment of Unstructured Grids for Detached-Eddy Simulation of High Reynolds Number Separated Flows," 8th ISGG Conference, Honolulu, June 2002.

27. Forsythe, J., Squires, K., Wurtzler, K., and Spalart, P., "Detached Eddy Simulation of Fighter Aircraft at High Alpha," AIAA Paper 2002-0591, Jan. 2002.
28. Pirzadeh, S., "Vortical Flow Prediction Using an Adaptive Unstructured Grid Method," Research \& Technology Organization Applied Vehicle Technology Panel Meeting, Norway, 7-11 May 2001.

29. Chan, W.M., Nash, S., Buning, P.G., and Rogers, S.E., "Chimera Grid Tools, Version 0.4," NASA Ames Research Center, Moffett Field, CA, Feb. 1998.

30. Tramel, R.W. and Suhs, N.E., "PEGSUS 4.0 User's Manual,” AEDC TR-91-8, June 1991.

31. Gursul, I., "Review of Unsteady Vortex Flows Over Delta Wings," AIAA Paper 2003-3942, June 2003. 